\title{
Uncertainty assessment of the road safety index
}

\author{
Elke Hermans ${ }^{\mathrm{a}, *}$, Filip Van den Bossche ${ }^{\mathrm{b}}$, Geert Wets ${ }^{\mathrm{a}}$ \\ a Transportation Research Institute, Hasselt University, Wetenschapspark 1 bus 12, 3590 Diepenbeek, Belgium \\ ${ }^{\mathrm{b}}$ European University College Brussels, EHSAL Centre for Economics and Management, Stormstraat 2, 1000 Brussels, Belgium
}

\section{A R T I C L E I N F O}

\section{Article history:}

Received 22 October 2007

Received in revised form

2 May 2008

Accepted 16 September 2008

Available online 8 October 2008

Keywords:

Road safety index

Ranking

Indicators

Weighting

Uncertainty

Sensitivity

\begin{abstract}
A B S T R A C T
Composite indicators aggregate domain-specific information in one index, on the basis of which countries can be assigned a relative ranking. Recently, the road safety community got convinced of the policy supporting role of indicators in terms of benchmarking, target setting and selection of measures. However, combining the information of a set of relevant risk indicators in one index presenting the whole picture turns out to be very challenging. In particular, the rank of a country can be largely influenced by the methodological choices made during the composite indicator building process. Decisions concerning the selection of indicators, the normalisation of the indicator values, the weighting of indicators and the way of aggregating can influence the final ranking. In this research, it is shown that the road safety ranking of countries differs significantly according to the selected weighting method, the expert choice and the set of indicators. From these three input factors, the selection of the set of indicators is most influential. A well considered selection of indicators will therefore establish the largest reduction in ranking uncertainty. With a set of appropriate indicators, the proposed framework reveals the major sources of uncertainty in the creation of a composite road safety indicator.
\end{abstract}

(c) 2008 Elsevier Ltd. All rights reserved.

\section{Introduction}

In recent years, the interest in and the use of indicators and indexes are rapidly increasing. In general terms, an indicator is a quantitative or a qualitative measure derived from a series of observed facts that can reveal relative positions (e.g. of a country) in a given area [1]. Their benefit for policy support and communication is a key advantage. Possible applications include trend identification, problem prediction, target and priority setting and impact assessment of measures [1,2]. In domains like economics (the human development index), technology (the technology achievement index) and sustainability (the environmental sustainability index) the aggregation of indicators in one index is common [3]. The most important advantage of one index over an accumulation of individual indicators is that all relevant information is aggregated in one final score which can be used for ranking countries, tracking changes over time, etc. However, the process of obtaining such an index is methodologically challenging.

In this research, we focus on the road safety domain, which is currently showing much interest in the use of indicators (e.g. [4]). Usually, road safety performance is compared over countries by means of the number of traffic fatalities per million inhabitants.

\footnotetext{
* Corresponding author. Tel.: +3211269141; fax: +3211269198.

E-mail address: elke.hermans@uhasselt.be (E. Hermans).
}

However, international accident data are characterised by various inconsistencies, like the lack of uniformity in definitions and the problem of under-registration [5]. The most important drawback is that knowledge of the number of accidents and casualties in a country is insufficient to understand the processes that lead to traffic accidents and casualties. To rank a set of countries according to their road safety level, the underlying risk factors and the road safety measures that determine the road safety performance should therefore also be taken into account. Road safety indicators are useful in this respect, as they offer a means to include these dimensions in the road safety ranking.

International research focused on the causes of road accidents to develop appropriate countermeasures. The relationship between numerous explanatory factors on the one handbehavioural, economic, climatologic, infrastructural, legislative, etc.-and the number of accidents and casualties on the other hand, has been studied amongst others by Hakim et al. [6], Scuffham [7], Eisenberg [8], Van den Bossche et al. [9] and Hermans et al. [10]. The most contributing road safety dimensions are the road user behaviour (speed, alcohol, seatbelt use), the vehicle safety (composition and age of the vehicle fleet) and environmental factors (expenditure on roads, gross domestic product, urban population, hospital care) [11,12].

For the most influential road safety risk factors, so-called safety performance indicators are presently being developed on the European level [4,13]. A safety performance indicator is defined as any measurement that is causally related to accidents 
and casualties; it is used in addition to accident and casualty counts in order to describe the safety performance of a country and to understand the process that leads to accidents [13]. Based on a set of carefully selected indicators, the safety situation in a country can be reflected, the impact of safety interventions can be measured and the safety performance of different countries can be compared [4]. Indicators can identify the domains that need additional policy efforts, and their aggregation into one road safety indicator offers a valuable tool for road safety assessment. Indeed, as a composite indicator can be computed for each country, the comparison with other countries allows identifying best practices and successful policies and can lead to optimal country-specific road safety actions. Also, because road safety risk information is available more quickly than official road safety statistics, measures can be taken to prevent increases in accidents and casualties.

Obviously, a sound methodology for constructing a road safety performance index is prerequisite for its use. To this end, a composite indicator methodology for road safety has to be elaborated. In Nardo et al. [1], 10 steps to create an aggregated index are discussed. The theoretical framework, the selection of indicators, the imputation of missing data, the normalisation, weighting and aggregation are all phases needed to develop an index. In the past, limited attention has been paid to the construction of a road safety index (for example [14]), and we believe that a methodologically valid composite indicator approach is a new, challenging and necessary concept in road safety. In order to develop an acceptable index, the subjective choices involved in the development of a road safety index need to be justified and their impact on the end result should be quantified. As there is no agreement or a priori knowledge on the best or ideal method for developing a composite indicator, several techniques for indicator selection, imputation, normalisation, weighting and aggregation need to be tested. Uncertainty and sensitivity analyses are a requirement for composite indicators. The end result-such as the ranking of countries based on their road safety index score-can be heavily influenced by the choices made in the index construction process. As stated in [15] the iterative use of uncertainty and sensitivity analysis contributes to the well structuring of the composite indicator, provides information concerning the robustness of the countries' ranking and identifies ways to reduce the uncertainty in the ranking for a better monitoring and policy.

Even though numerous composite indicators have been developed in several domains, only for some of them the uncertainty and sensitivity of the results have been tested. A comprehensive description of uncertainty analysis (UA) and sensitivity analysis (SA) is given in [16]. In this study several aspects of the index construction process are involved and the theory is applied to the technology achievement index. The SA indicated the most influencing factors. For the Internal Market Index [17] the effect of different weights is studied. For each of the 12 indicators the frequency of the weights given by all experts was calculated from which a sample of 5000 random points was generated. The SA highlighted the three indicators for which more consensuses on their relative merit should be aimed at. The e-business readiness composite indicator developed by Tarantola et al. [18] assesses the preparedness of the internet business environment of European countries. The influence of the variability in the weights and the uncertainty in the imputed data on the composite indicator is studied. The analysis is carried out independently for each country.

The remaining of this paper is structured as follows: in Section 2 the design of this study is described. Section 3 focuses on the methodology. The theoretical considerations of uncertainty and sensitivity analysis are briefly discussed. Subsequently, the results of this research are shown in Section 4. This paper closes with the main conclusions and some topics for further research in Section 5.

\section{Study design}

The aim of our research is to obtain a measurement of the uncertainty and sensitivity in a road safety ranking that is caused by various choices made during the construction process of a composite road safety indicator. To this respect, we will use several methods in each step of this process. In [1,3] a number of often used and relevant methods for imputation, normalisation, weighting and aggregation are given. In our study, we will investigate the impact of the weighting method, the expert choice and the indicator selection on the road safety ranking of countries. In this section we discuss the indicator selection procedure and the development of the theoretical framework.

\subsection{Selection of road safety indicators}

As road safety is typically characterised by a lack of accidents and victims, the magnitude of the problem is usually measured by the opposite, i.e. the level of road unsafety. The selection of road unsafety indicators follows from a number of steps. First, road unsafety is quantified by means of the number of accidents and casualties. Next, the domains which contribute to either accidents, casualties or both are listed. As stated before, in literature there is agreement on a group of relevant risk factors. The European SafetyNet project [4] on safety performance indicators has selected alcohol and drugs, speed, protective systems, visibility, vehicle, infrastructure and trauma management as crucial road safety risk domains. In theory, each risk domain should be characterised by a set of indicators. Which indicators to select from the set of possible candidates can be determined by means of selection criteria (see e.g. [1,2]). Additionally, multivariate data analysis techniques could be helpful during the selection process. However, for the road safety risk domains, the number of indicators is limited, and the selection process is mainly driven by data availability. In order to assess the relative road safety performance of as much countries as possible, one indicator per domain will be used. When selecting the indicators, it was ensured that the criteria of policy relevance and clarity were satisfied in addition to available, reliable and comparable data.

The three most important behavioural risk factors of road safety are alcohol, speed and protective systems. A larger blood alcohol concentration (BAC) implies a higher probability of getting involved in an accident [12]. Moreover, consumption of alcohol has been seen to increase the frequency of fatal accidents [6]. The percentage of road users driving under the influence of alcohol (i.e. having a BAC above the legal limit) is the chosen indicator. Secondly, inappropriate or excessive speed has been identified as a highly important factor influencing both the frequency and severity of accidents [5]. The speed indicator in this study measures the share of drivers exceeding the speed limit on a particular road type (highways in this case). For the protective systems domain, the seatbelt wearing rate in front is the selected indicator. Protective systems in general and seatbelts in particular reduce the risk of fatal and serious injury [12]. Next, many accidents occur because road users do not notice each other in time, even in daylight [4]. Vehicle visibility is therefore one of the factors affecting road safety. The visibility domain focuses on daytime running lights (DRL). As a proxy, a categorical indicator specifying whether a DRL law is in place (value 3 ) or not (value 1 ) is chosen. Value 2 is assigned for a partial DRL law obligating the 
use of daytime running lights for specific vehicle categories, road types or time periods.

The vehicle indicator using the age of the vehicle fleet (or a particular share) indicates the level of protection that the vehicle gives to its occupants. Newer cars are equipped with more advanced technologies assisting in accident avoidance and severity reduction of accident outcomes [12]. Next, the infrastructure domain is one for which several indicators can be thought of but for which currently very few data are available [13]. Therefore, the network density, defined as the network length divided by the area, has been chosen as indicator where a more dense road network is in our opinion linked to some accessibility and safety enhancing infrastructural expenses. Finally, trauma care refers to the system responsible for the medical treatment of injured persons from a road accident. The probability of surviving and the quality of life after the accident are influenced by the level of trauma care [13]. The number of hospitals per 1000 inhabitants is a proxy for trauma care. More hospitals imply a faster service, which has a positive influence on the level of injury.

To enhance the interpretation of the results, the alcohol and speed indicators have been transformed such that for all indicators a lower value always implies more unsafety. As a result, a country should try to maximize its indicator values. The seven indicators are presented in Table 1. From the summary statistics, it can be seen that the alcohol limit is respected more than the speed limit; there are large differences in seatbelt wearing across the countries; the average share of young (i.e. $<6$ years) cars is $37 \%$ while there are on average 4 hospitals per 1000 inhabitants. The seven indicator values are known for the 18 European countries in our data set leaving the imputation of missing values unhandled in this study. The data have been standardised to compute the composite values.

\subsection{Theoretical framework}

An overall theoretical framework on the road safety performance index is shown in Fig. 1. On the left, the risk domains and selected performance indicators are presented. Next, the major steps involved in the construction of a composite index are given. All (collected and imputed) indicator values need to be normalised and subsequently weighted and aggregated. As subjective choices are often made during the construction process, the uncertainty and sensitivity analysis is an essential and iterative step that directs the methodological process and eventually results in a robust road safety index. Finally, for each country an

Table 1

Summary statistics on the indicator data.

\begin{tabular}{|c|c|c|c|c|c|c|c|}
\hline & Alcohol & Speed & $\begin{array}{l}\text { Protective } \\
\text { systems }\end{array}$ & Visibility & Vehicle & Infrastructure & $\begin{array}{l}\text { Trauma } \\
\text { care }\end{array}$ \\
\hline Maximum & 100.0 & 90.0 & 97.0 & 3.0 & 61.0 & 4.5 & 6.3 \\
\hline Minimum & 92.1 & 54.0 & 40.0 & 1.0 & 17.0 & 0.3 & 2.2 \\
\hline Mean & 97.1 & 74.6 & 79.4 & 2.1 & 37.4 & 1.7 & 4.2 \\
\hline Variance & 5.5 & 109.2 & 196.8 & 0.5 & 129.1 & 1.4 & 1.8 \\
\hline
\end{tabular}

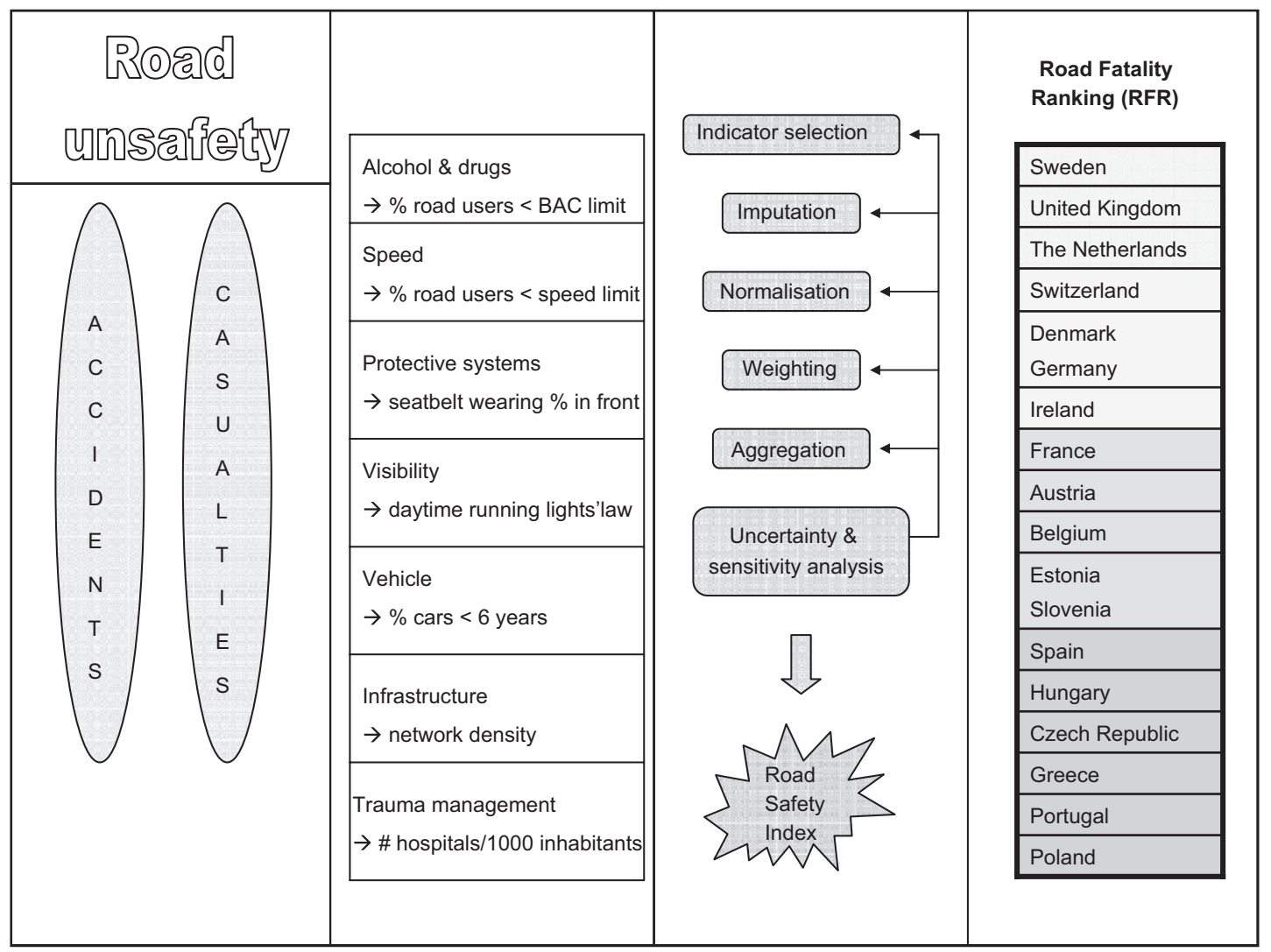

Fig. 1. Framework of the road safety performance index. 
index score is obtained based on which a ranking can be provided. The last column in Fig. 1 presents a possible, often used ranking which makes use of the number of road fatalities per million inhabitants in 18 European countries (in 2003). Notice that this ranking is based on just one outcome indicator and not on a performance index. Sweden, the United Kingdom and the Netherlands are the safest countries in this road fatality ranking (RFR).

To illustrate the importance and implication of an uncertainty and sensitivity analysis in this theoretical framework, three methodological aspects will be discussed in this paper. We will consider two weighting methods, incorporate the opinion of several experts and assess the impact of the indicator/domain selection on the ranking of the 18 countries in our data set.

More specifically, we will test two commonly used weighting methods [3] both based on expert opinions, namely the analytic hierarchy process (AHP) and budget allocation (BA). AHP developed by Saty [19] is a comprehensible and valuable technique for assessing indicator weights. For each pair of indicators, an expert is asked to indicate which of the two is contributing more to road unsafety and how large the intensity of the difference is. This expert information is presented in a matrix from which the indicator weights and the degree of consistency are calculated using the eigenvector technique. BA is another well known method for obtaining indicator weights. A number of experts are asked to distribute a given budget over the indicators in such a way that spending more on an indicator implies that (s)he wants to stress its importance [1]. The share of the budget assigned to each indicator determines its weight. The results of comparisons in pairs of the road safety indicators as well as the allocation of a budget over the indicator set were obtained from nine road safety experts familiar with the causes and risk domains of road safety. All experts participating in a European road safety project were asked to cooperate and as they originated from different European countries (Czech Republic, France, Greece, Hungary, Israel, the Netherlands, Norway, Switzerland and the United Kingdom) an international perspective was obtained. They gave their opinion independently from each other. The main statistics on the AHP and BA set of weights are given in Table 2. It can be seen from the table that on average, alcohol and speed receive most weight from the experts while visibility gets the least weight. The range (i.e. the difference between the maximum and minimum value) and the variance indicate some level of disagreement. Moreover, the two weighting methods result in a discrepancy in the average weight for trauma care. As a safe option, the average indicator weights over the experts are often used to construct the index [19]. However, we will consider all available, valuable information and assess the impact of selecting the weights from one particular expert under each of the two methods. In addition, the countries' ranking will be studied for alternative sets of six or seven indicators. As we aim for simplicity in the development of our index, we have to reconsider the selected domains (and indicators) during the process.

In general, the results of the uncertainty and sensitivity analysis will indicate how robust the ranking is, which of these methodological choices has the largest impact on the absolute average rank shift and which input factor needs special effort in order to reduce the uncertainty. As in other research (e.g. [15]) the output variable of interest in this study is the average change in the countries' rankings for all possible scenarios. Yet, due to the specific nature of this composite indicator we will compare the road safety ranking against the reference ranking based on the number of traffic fatalities per million inhabitants. In addition, an analysis on the country level will be performed to show which countries have the largest change in rank under the different methodological options.

\section{Method}

\subsection{Introduction}

Uncertainty analysis and sensitivity analysis are terms that often appear in the context of models. Although it seems that everyone agrees on the importance of these analyses, in practice this step is often lacking. Similar to for example the confidence interval around the regression coefficient of a particular explanatory variable, a confidence interval should be added to the position of a country in a ranking. Even though the relative position of a country is very appealing for communication purposes, there is the danger that attention is paid to this number only without considering the uncertainty that is created by the methodological assumptions that were made during the construction process and the sensitivity of the final ranking to these assumptions. Obviously, UA and SA are essential steps in the context of composite indicators and ranking. As the number of different methodological choices in the several steps of the index construction process is substantial, the end result can be manipulated relatively easily. After the selected methods have been applied to the data set, it is good practice to interpret the final results by means of an uncertainty and sensitivity analysis. This information can be used to construct a more robust index which will be better accepted.

\subsection{Description of the uncertainty and sensitivity analysis}

In general, uncertainty analysis estimates the uncertainty in the output taking into account the uncertainty affecting the input

Table 2

Summary statistics on the expert weights.

\begin{tabular}{|c|c|c|c|c|c|c|c|}
\hline & Alcohol & Speed & $\begin{array}{l}\text { Protective } \\
\text { systems }\end{array}$ & Visibility & Vehicle & Infrastructure & $\begin{array}{l}\text { Trauma } \\
\text { care }\end{array}$ \\
\hline \multicolumn{8}{|l|}{ AHP } \\
\hline Maximum & 0.373 & 0.458 & 0.385 & 0.029 & 0.207 & 0.340 & 0.180 \\
\hline Minimum & 0.117 & 0.144 & 0.055 & 0.014 & 0.031 & 0.033 & 0.030 \\
\hline Mean & 0.259 & 0.291 & 0.143 & 0.024 & 0.086 & 0.113 & 0.085 \\
\hline Variance & 0.008 & 0.010 & 0.010 & 0.000 & 0.005 & 0.010 & 0.002 \\
\hline \multicolumn{8}{|l|}{$B A$} \\
\hline Maximum & 0.357 & 0.429 & 0.357 & 0.071 & 0.143 & 0.286 & 0.243 \\
\hline Minimum & 0.071 & 0.143 & 0.071 & 0.000 & 0.014 & 0.014 & 0.029 \\
\hline Mean & 0.246 & 0.294 & 0.125 & 0.041 & 0.068 & 0.102 & 0.125 \\
\hline Variance & 0.010 & 0.013 & 0.008 & 0.001 & 0.001 & 0.009 & 0.006 \\
\hline
\end{tabular}


factors. Rather than being a unique value the estimated output represents a distribution of values and elementary statistics such as the mean, standard deviation and percentiles are used to describe its features [20]. At the same time, sensitivity analysis is defined as the study of how uncertainty in the output of a model can be apportioned to different sources of uncertainty in the model input. Saltelli et al. [20] present a step-by-step plan for performing a sensitivity analysis on a model:

1. The goal of the analysis and consequently the form of the output function need to be defined. The output of interest is a single quantity whose value is the top-most information that the model is supposed to provide.

In the present study, the obtained rank of each country is compared with the road fatality rank of the country and the absolute average over these 18 values is the output of interest. It captures the absolute average shift in rank of the entire set of countries relative to the ranking based on the number of road fatalities per million inhabitants. A higher shift indicates a larger uncertainty.

2. A decision has to be made on which input factors to include in the sensitivity analysis.

Here, three input factors will be incorporated, making use of triggers to decide on one of the possible scenarios. The first input factor selects between the AHP and BA weighting method. The trigger for the second input factor is used to select one of the nine experts. Thirdly, it needs to be determined if all seven indicators are considered or six indicators (there are eight alternatives for this input factor).

3. For each input factor, a distribution function has to be chosen. In addition, a correlation structure between input factors can be defined if appropriate.

All input factors in our study are uniformly distributed between values 1 and 3 for the first input factor, between 1 and 10 for the second input factor and between 1 and 9 for the third input factor.

4. A sensitivity analysis method must be chosen based on the following three aspects: the question that needs to be addressed, the number of model evaluations (which has to be affordable in terms of model execution time) and the presence of a correlation structure between input factors.

We will apply the method of Sobol (more information is given in Section 3.3).

5. The input sample is generated.

Using the selected method, an $N \times 3$ input sample is produced. For each of the three input factors $N$ (= several thousands) values are drawn from their probability distribution function.

6. The model needs to be elaborated on the generated sample and the output produced.

The three values in each row of the sample need to be translated in one output score. First, the values need to be converted to make clear which weighting method, which expert and which indicators will be used in the construction of the index. The composite indicator value for all countries in the data set results from the weighted sum of the road safety indicators. Based on their score, each country is assigned a rank number between 1 and 18. The output value is the average of the absolute value difference between the road fatality rank number of each country and its rank number based on the composite indicator score. $N$ output values are obtained.

7. The model output is finally analysed and conclusions are drawn.

The empirical probability distribution function of the output can be estimated which determines the uncertainty of the output resulting from the uncertainty in the input. As sensitivity analysis results some indices are computed (see in Section 3.4).

\subsection{Global variance-based Sobol method}

A wide range of methods for sensitivity analysis can be found in literature. First, a distinction can be made between local and global methods. As local methods explore only one point of the factor's space and factors are changed one at a time, a global analysis is more appropriate in this case. Global methods explore the entire interval of each factor and the effect for a factor is the average over the possible values of the other factors. In recent years global quantitative sensitivity analysis techniques have received considerable attention in literature [20].

One important and promising class of sensitivity methods is referred to as variance-based techniques. Their characteristic of being model-free (i.e. applicable to non-linear and non-additive models) makes them very useful for composite indicators, in which several layers of uncertainty are simultaneously present [1]. Variance-based techniques have a number of advantages [20]: they can explore the whole range of variation of each input factor instead of sampling the factors over a restricted number of values, they are able to capture interaction effects apart from the fractional contribution of input factor $X_{i}$ to the variance of the model output $Y$, are easy to interpret and explain, are quantitative, etc.

In this study we use the method of Sobol for generating the input sample and obtaining uncertainty and sensitivity results. This variance-based method is a well known, often used method with useful characteristics as it requires no seed (and hence this has no impact) and handles discontinuities well.

\subsection{Technical considerations of sensitivity analysis indices}

Saltelli et al. [20] provide detailed information on sensitivity indices. $S_{i}$ is the first-order effect of input factor $X_{i}$ on output $Y$. Synonyms are importance measure, correlation ratio or sensitivity index. The total effect terms $S_{T i}$ give information on the total effect of input factor $X_{i}$ on output $Y$. The main aspects of the first-order and total effect sensitivity indices are shown in Table 3. Saltelli et al. [20] state that by computing all $S_{i}$ and $S_{T i}$ terms, a fairly complete and parsimonious description of the model can be obtained in terms of its global sensitivity properties.

\section{Analysis and results}

Following the step-by-step plan discussed in Section 3.2, an uncertainty and sensitivity analysis is performed on our road safety data set. First, the three uncertain input factors are described. More specifically, a uniform probability distribution function is assigned to the weighting system (AHP or BA), the expert selection (experts 1-9) and the indicator set (7 or 6 road safety indicators). Next, from these distributions values are drawn using the Sobol technique. The number of runs for this model is a factor $2^{k}$ of $256(k=0,1,2, \ldots)$. As we want to draw conclusions based on several thousands of runs, a sample matrix $M$ consisting of $N(=8192)$ rows and $F(=3)$ columns is generated. ${ }^{1}$ Subsequently, each of the 8192 rows of our generated sample is

\footnotetext{
${ }^{1}$ It should be noted that due to the study design - with discrete input factors and a low number of options (i.e. 144 combinations in total)-the number of sampling runs undertaken (i.e. 8192) exceeds the number of runs actually needed However, in this way we anticipate future extensions of the road safety index methodology including other input factors such as imputation. The Sobol method discussed in this study is still valid then.
} 
Table 3

Summary information on first-order and total effect sensitivity indices.

\begin{tabular}{|c|c|}
\hline$S_{i}=\frac{V\left(E\left(Y \mid X_{i}\right)\right)}{V(Y)}$ & $S_{i}$ indicates how much on average the output variance can be reduced if $X_{i}$ is fixed \\
\hline$S_{i}=$ high & $X_{i}$ is an influencing input factor which drives the model output variance and deserves future effort to reduce the uncertainty \\
\hline$S_{T i}=\frac{E\left(V\left(Y \mid X_{-i}\right)\right)}{V(Y)}$ & $S_{T i}$ captures the total effect of $X_{i}$ on $Y$ \\
\hline $\begin{array}{l}S_{T i}-S_{i} \\
S_{T i}=0 \\
\sum_{i} S_{T i}>1 \text { or equivalent } \sum_{i} S_{i}<1\end{array}$ & $\begin{array}{l}\text { This measure indicates how much } X_{i} \text { is involved in interactions with other input factors } \\
X_{i} \text { is non-influential } \\
\text { Interactions exist between the factors }\end{array}$ \\
\hline
\end{tabular}

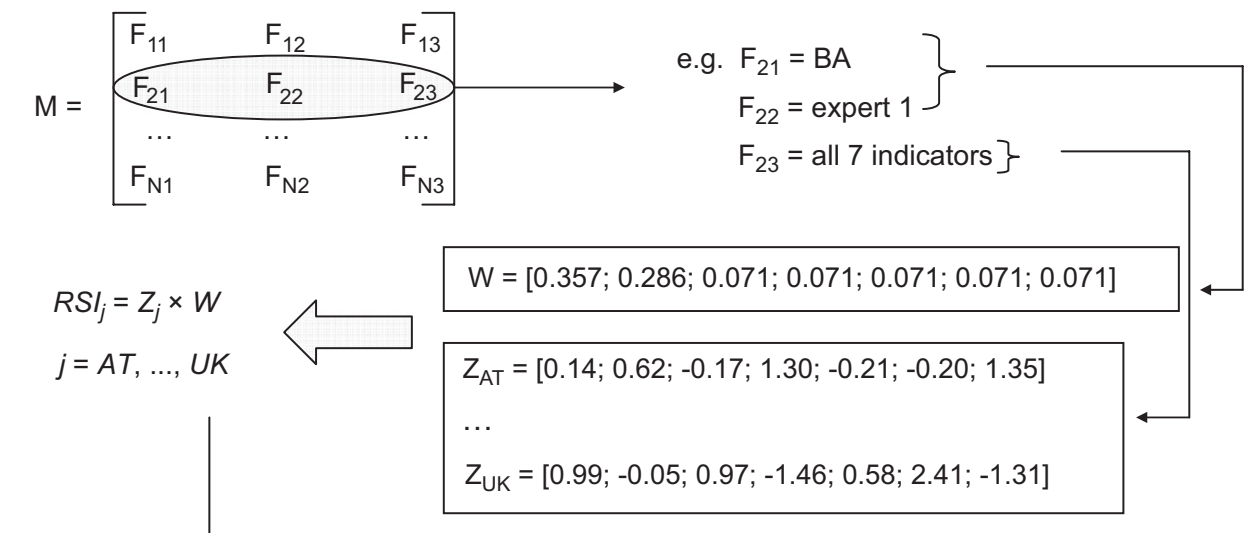

$$
\begin{aligned}
& \begin{aligned}
\square & Y=\frac{1}{18} \sum_{j=1}^{18}\left|R S R_{j}-R F R_{j}\right| \\
Y & =\frac{1}{18}(2+\ldots+2)=\underset{\text { for row } 2}{5.72}
\end{aligned}
\end{aligned}
$$

\begin{tabular}{|c|c|c|}
\hline Country & Order of rank RSI & Road fatality ranking \\
\hline AT & 7 & 9 \\
\hline$\ldots$ & $\ldots$ & $\ldots$ \\
\hline UK & 4 & 2 \\
\hline
\end{tabular}

Fig. 2. Scheme describing the assessment of the output of interest.

evaluated to obtain a score for the output of interest. As we make use of triggers to select one of the options for each factor, the sampled values need to be translated in a set of weights (based on the weighting method and the chosen expert) and (standardised) indicator values to calculate the composite road safety index scores for the 18 countries. This working method is illustrated in Fig. 2 for the second row of sample $M$.

Next, a road safety rank (RSR) is assigned to the countries based on their road safety index score (a higher index score implies a better rank). Using the road fatality ranking of the 18 countries (resulting from the number of road fatalities per million inhabitants) the difference between the two rank numbers is calculated for each country. Subsequently, the average of these 18 absolute values is taken resulting in the output value for this row. This process is repeated until $N$ output values are obtained.

The $N$ output values are used to obtain the uncertainty and sensitivity outcomes. The uncertainty analysis results in the output distribution over the sample. As shown by the histogram in Fig. 3(a), the absolute average shift in ranking over all 18 countries compared with the road fatality ranking has an average of 5.64 positions with a variance of 0.78 . A detailed analysis of the results shows that the largest absolute average shift in country ranking occurs in case the composite indicator consists of the weights assigned by expert 6 using the budget allocation method and six road safety indicators (i.e. the infrastructure indicator is no longer part of the data set). Due to the specific nature of our data, we already expected quite large differences between the two rankings. Although we aim to describe the road safety performance of countries by means of relevant indicators, there still may be some discrepancy with the ranking based on road traffic fatalities. Some indicators may not have a causal relationship with the number of traffic fatalities, we probably need to incorporate more road safety indicators, some indicators may only be relevant for a certain group of countries or the quality of the data may be rather poor. However, the road fatality ranking offers valuable information and is an appropriate ranking to compare our composite indicator ranking to. Secondly, we use a rather small data set of European countries for which a change of a few ranks is more likely to happen than in case of a larger set of countries on a world scale having a more distinct motorization and road safety level.

Fig. 3(b) shows the disaggregation of the global absolute average shift in rank. For each country the average over 8192 values of the absolute difference between their rank based on their road safety index score and their road fatality rank is shown in increasing order. The road safety index score for Poland results in a better road safety rank than its 18th place in the road fatality ranking. A detailed look at the data shows that Poland scores relatively well on alcohol and speed and to these indicators above-average weights are mostly assigned by the experts. This large discrepancy for Poland influences the global 
a

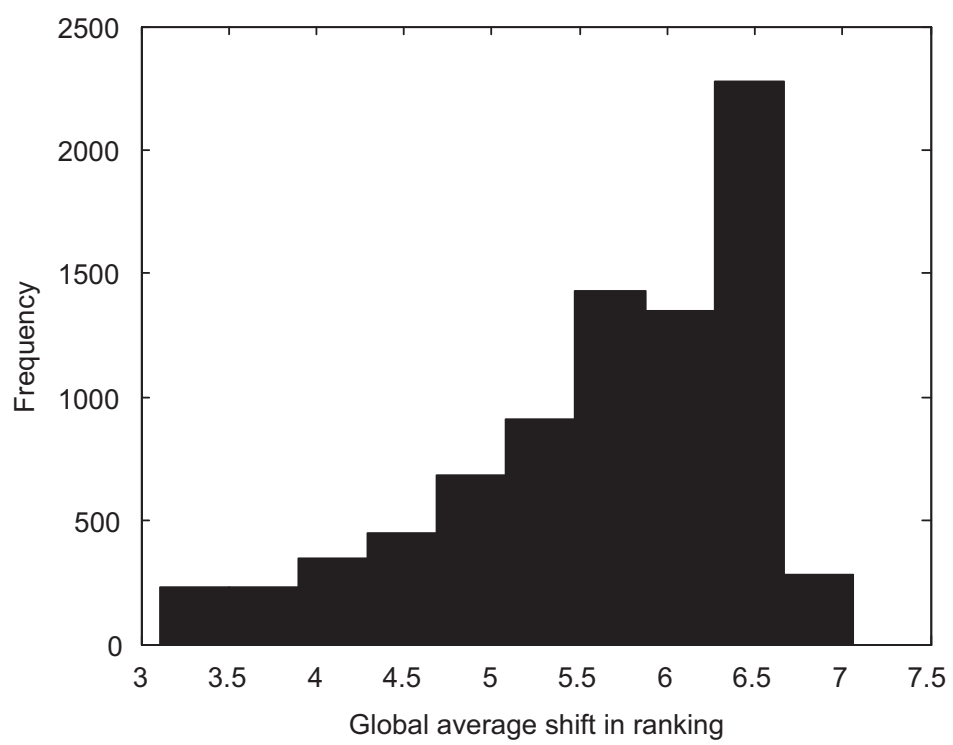

b

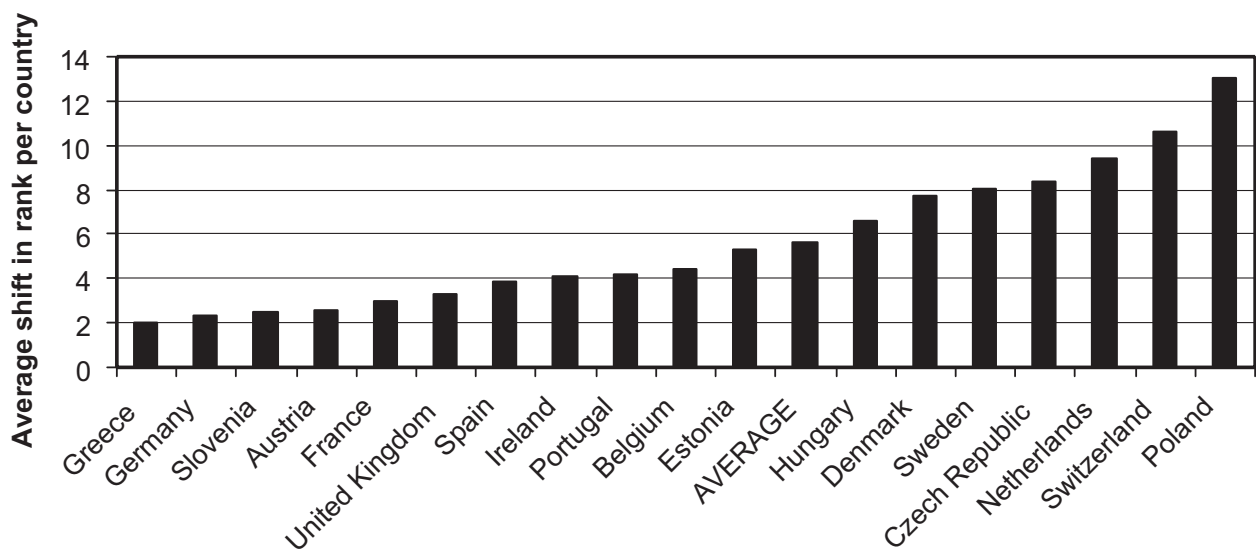

C

\begin{tabular}{|l|c|c|}
\hline & First-order sensitivity index & Total effect sensitivity index \\
\hline Indicator selection & 0.508 & 0.639 \\
\hline Weighting method & 0.044 & 0.131 \\
\hline Expert selection & 0.300 & 0.467 \\
\hline Non-additivity & 0.148 & \\
\hline
\end{tabular}

Fig. 3. Uncertainty and sensitivity results.

absolute average shift. The methodological choices in this study only slightly affect the rank number of countries like Greece and Germany.

Finally, the sensitivity analysis calculates the first-order index $S_{i}$ for each of the three input factors, which captures the fractional contribution to the model output variance due to the uncertainty in $X_{i}$ and the total effect index $S_{\mathrm{T} i}$, which concentrates all the interactions involving factor $X_{i}$ in one single term. As shown by the numbers in Fig. 3(c) the set of road safety indicators has a very high first-order index (0.508). Additionally, the index of expert selection (0.300) is also relatively high. Consequently, the sum of the three first-order sensitivity indices-0.852-approaches one, meaning that the model is not totally additive and interactions emerge between the input factors. Therefore, the calculation of total effect sensitivity indices is justified. The relative differences between the total effect and first-order sensitivity indexes indicate that the factor expert selection accounts for most of the interaction effects (0.167).

To conclude, the selection of the set of indicators is the most influencing input factor. The uncertainty in the absolute average shift in countries' ranking could be reduced most if more effort was directed in this topic. In case best needed (i.e. the most ideal) indicators instead of best available indicators could be used, the influence of the indicator selection factor would probably be reduced. The impact of the choice of the expert to assign the weights is also significant. The weighting 
method (analytic hierarchy process or budget allocation) is the least important factor with limited interaction effects with the other input factors. In other composite indicator studies (cf. Section 1) the uncertainty and sensitivity analysis resulted in more or less the same results. Nardo et al. [15] tested normalisation, indicator selection, aggregation, weighting, expert selection and imputation for the technology achievement index and concluded that the indicator selection, the weighting method and the choice of the expert were the most important factors. The sensitivity results of our study indicate that these aspects need further attention in the development of a road safety index as well.

\section{Conclusions and further research}

The development of a road safety index is a challenging and necessary task. Offering policymakers a useful tool for ranking and benchmarking countries, this road safety index can help enhancing the understanding of the road safety problem. However, the process of constructing such an index is complex and consists of several phases. In each phase, the researcher is left with methodological choices that might influence the final ranking. In literature, there is no agreement on which method or technique to use in each phase. As a result, one method is often chosen (at random or based on simplicity) and the index score or country ranking is presented and used without considering the assumptions made during the process. Founding a policy on subjective choices is inappropriate. In order to provide a robust, methodologically sound index, uncertainty and sensitivity analyses are a prerequisite.

In this study, an uncertainty and sensitivity analysis has been elaborated. We examined the impact of three aspects (the selection of the weighting scheme, the expert and the indicator set) on the absolute average shift in countries' ranking based on the road safety index compared with the road fatality ranking. Based on the results of this study a number of road safety enhancing recommendations can be formulated. First, it has been shown by the uncertainty analysis that the global absolute average shift in ranking based on the road safety index compared with the road fatality ranking of the 18 countries in our data set equals 5.6 positions. Rankings receive a lot of attention from policymakers as well as the public. Since an absolute average country shift in rank between 2 and 13 implies that a particular country can be ranked either very high or very low, it is required to always assess the impact of methodological choices on the ranking of countries. We therefore recommend the frequent use of uncertainty analysis as essential part of the methodology of the road safety index development process.

The sensitivity analysis answered the question which of the uncertain input factors is most influential. From literature, it already appeared that the factors studied here are all very important.

The set of indicators that is used to construct the road safety index seems to have the largest impact on a shift in ranking. Therefore, the theoretical framework of a road safety performance index needs some careful thought. More specifically, the risk domains of road unsafety need to be identified and attention should be paid mainly to the indicators describing these domains. In this study, the seven risk domains defined in the SafetyNet project [4] were used and one indicator for each domain was selected (based on data availability, policy relevance and clarity). Of the seven indicators in the present study, a data set without the protective systems indicator, without the infrastructure indicator or without the alcohol indicator caused the highest global shift in countries' ranking. In the future, more indicators will be considered and their influence explored in order to give a complete picture of road safety. The sensitivity results can then be used to assess whether the selected indicators are successful in reducing the rank uncertainty for the countries under study.

Besides the indicators the road safety index consists of a set of indicator weights. Two factors were used in this respect: one selecting the weighting method (analytic hierarchy process or budget allocation) and other deciding on the expert ( 1 of the 9 experts). The analysis showed that the expert selection (more than the weighting method) had an impact on the shift in countries' ranking (singularly as well as by means of interaction effects). Therefore, the selection of experts is crucial and needs careful thought. A group of experts with an excellent knowledge and experience in road safety but at the same time a specific view and background has to be found. The weighting method is the factor with the least impact. However, this result is caused by the similarity between the two alternatives. AHP and BA belong to the same family of participatory methods which possibly makes the choice between them of lesser impact. In the future the weighting method should be considered in a broader sense. Other relevant weighting techniques for the road safety context such as data envelopment analysis [21] should be incorporated in the model as the choice between several weighting methods may influence the end result to a large extent.

This paper showed the advantages of an uncertainty and sensitivity analysis for a road safety composite indicator by means of a case study. As the road safety performance index is still under development, several topics will be further investigated. Other development aspects like aggregation, normalisation and imputation of missing values should be incorporated in the analysis as well. The complexity of the model can be reduced in case the least influential inputs are identified and subsequently fixed to an acceptable technique. Furthermore, a more detailed analysis on country level could identify the assumptions under which each country performs best. The uncertainty and sensitivity results, e.g. an ordering of all input factors in terms of importance, can be used to make sound decisions on certain methodological aspects. That way, the added value of the road safety index together with its acceptance and use as a valuable policy support tool will increase.

\section{References}

[1] Nardo M, Saisana M, Saltelli A, Tarantola S, Hoffman A, Giovannini E. Handbook on constructing composite indicators: methodology and user guide. STD/DOC(2005)3, OECD, 2005.

[2] Litman T. Developing indicators for comprehensive and sustainable transport planning. In: Proceedings of the 86th annual meeting of the Transportation Research Board, Washington, DC, 2007.

[3] Saisana M, Tarantola S. State-of-the-art report on current methodologies and practices for composite indicator development. EUR20408EN, EC-JRC, 2002.

[4] SafetyNet. State of the art report on road safety performance indicators. D3.1, 2005.

[5] Evans L. Traffic safety. Bloomfield Hills, MI, 2004.

[6] Hakim S, Shefer D, Hakkert AS, Hocherman I. A critical review of macro models for road accidents. Accid Anal Prev 1991;23:379-400.

[7] Scuffham PA. An econometric analysis of motor vehicle traffic crashes and macroeconomic factors. PhD. thesis, Otago University, New Zealand, 2001.

[8] Eisenberg D. The mixed effects of precipitation on traffic crashes. Accid Anal Prev 2004;36:637-47.

[9] Van den Bossche F, Wets G, Brijs T. Analysis of road risk per age and gender category: time series approach. In: Proceeding of the 86th annual meeting of the Transportation Research Board, Washington, DC, 2007.

[10] Hermans E, Van den Bossche F, Wets G. The frequency and severity of road traffic accidents studied by state space methods. J Transp Stat 2006;9: 63-76.

[11] Al Haji G. Towards a road safety development index. PhD. thesis, Linköpings University, Sweden, 2005.

[12] World Health Organization. World report on road traffic injury prevention 〈http://ww.who.int/world-health-day/2004/infomaterials/world_report/en 〉, 2004. 
[13] European Transport Safety Council. Transport safety performance indicators. ETSC, 2001.

[14] Al-Haji G. Road safety development index (RSDI). In: Proceedings of the 16th ICTCT workshop, Soesterberg, 2003.

[15] Nardo M, Saisana M, Saltelli A, Tarantola S. State-of-the-art report on simulation and indicators. D7.1, JRC, 2005.

[16] Saisana M, Saltelli A, Tarantola S. Uncertainty and sensitivity analysis techniques as tools for the analysis and validation of composite indicators. J R Stat Soc A 2005;168(2):307-23.

[17] Tarantola S, Saisana M, Saltelli A. Internal market index 2002: technical details of the methodology. JRC; 2002.

[18] Tarantola S, Nardo M, Saisana M, Gatelli D. A new estimator for sensitivity analysis of model output: an application to the e-business readiness composite indicator. Reliab Eng Syst Saf 2006;91:1135-41.

[19] Saaty TL. The analytic hierarchy process. New York, USA: McGraw-Hill; 1980.

[20] Saltelli A, Ratto M, Andres T, Campolongo F, Cariboni J, Gatelli D, et al. Global sensitivity analysis. The Primer, Wiley; 2008.

[21] Hermans E, Van den Bossche F, Wets G. Combining road safety information in a performance index. Accid Anal Prev 2008;40:1337-44.

Elke Hermans graduated in 2004 at Hasselt University, Belgium as Master of Business Economics. In her dissertation entitled "Road safety over time: an explorative research" the trend in the number of accidents and casualties in Belgium during the last decennia was modeled by means of state space models. Since October 2004 she works for the Transportation Research Institute IMOB of Hasselt University as a Ph.D. researcher. She is doing research in the area of road safety. More specifically, her research deals with the creation of a framework for road safety indicators. She has published and presented her research in a number of journals and at international conferences.

Filip Van den Bossche graduated in 1998 as Commercial Engineer in Management Informatics and Qualified Teacher in Economics and Applied Economics at the Catholic University of Leuven, Belgium. At the same university, he then obtained the degrees of Master of Science in Applied Economics (1999) and Master in Statistics (2002). After a first work experience as credit scoring analyst and consultant analyst, he started working at the Transportation Research Institute of Hasselt University, Belgium in 2001 as a doctoral researcher. He obtained his doctoral degree in 2006. In 2007, he started working at the European University College, Brussels. His research interest lies in the application of advanced time series analysis techniques. In this area, he is author and co-author of various research reports and articles.

Geert Wets received a degree as Commercial Engineer in Business Informatics from the Catholic University of Leuven, Belgium in 1991 and a Ph.D. from Eindhoven University of Technology, The Netherlands in 1998. Currently, he is an Associate Professor at the Faculty of Applied Economics at Hasselt University, Belgium, where he is Director of the Transportation Research Institute IMOB. His current research entails traffic safety modeling, transportation modeling and data mining. He has published his research in several international journals such as Accident Analysis and Prevention, Environment and Planning, Geographica Analysis, Knowledge Discovery and Data Mining, Transportation Research Record, and Information Systems. 